\title{
Timpanoplastías en el Hospital San Vicente de Arauco: Revisión de 3 años
}

Tympanoplasties in San Vicente de Arauco Hospital: 3 years review

\author{
Gonzalo Ortega F. ${ }^{1}$, Griselda Cordero L. ${ }^{1}$, Valeria Godoy R. ${ }^{2}$
}

${ }^{1}$ Hospital San Vicente de Arauco. Arauco, Chile.

${ }^{2}$ Hospital Kalvu Llanka. Cañete, Chile.

Los autores declaran no presentar conflictos de interés.

Recibido el 29 de septiembre de 2020. Aceptado el 20 de marzo de 2021

Correspondencia: Gonzalo Ortega F.

Chacabuco 827

Concepción, Chile

Email: gonzaloorteg@gmail.

com

\section{Resumen}

Introducción: La timpanoplastía es el tratamiento de elección en la otitis media crónica simple. En Chile se han realizado diversos estudios para evaluar el éxito de la timpanoplastía. En nuestro centro evaluamos el resultado anatómico y funcional, correlacionando los resultados con la demografía de nuestra población. Objetivo: Realizar una descripción epidemiológica de los pacientes con diagnóstico de otitis media crónica simple, sometidos a timpanoplastía en el Hospital San Vicente de Arauco entre los años 2017 y 2019. Material y Método: Estudio retrospectivo y descriptivo. Se revisaron las fichas clínicas de pacientes con cirugía realizada entre enero de 2017 y noviembre de 2019. Este estudio cuenta con la aprobación del comité de ética del servicio de salud Arauco. Resultados: Se realizaron 77 timpanoplastías. 56 oídos cumplieron los criterios de inclusión. 71,43\% fueron de sexo femenino. El rango de edad es de 8 a 64 años. $92,86 \%$ corresponde a timpanoplastía tipo I. Se utilizó un abordaje endoauricular en el 58,93\%. El injerto utilizado fue predominantemente compuesto (cartílago-pericondrio) en un $75 \%$. Se usó una técnica medial en un $94,94 \%$. Se obtuvo un éxito anatómico $85,71 \%$ de los pacientes. Se obtuvo una ganancia sobre $10 \mathrm{~dB}$ en un $60,71 \%$ de los pacientes. Conclusión: No se encontraron diferencias significativas importantes que relacionan las elecciones quirúrgicas con los resultados anatómicos y auditivos del procedimiento a mediano plazo.

Palabras clave: Otitis media crónica, timpanoplastía, perforación timpánica.

\begin{abstract}
Introduction: Tympanoplasty is the treatment of choice for chronic otitis media. In Chile, several studies have been carried out to evaluate the success of tympanoplasty. Here, we assesed the anatomical and functional outcomes, correlating the results with the demographics of our population. Aim: To carry out an epidemiological description of patients with a diagnosis of chronic otitis media who underwent tympanoplasty at our hospital between 2017 and 2019. Material and Method: Retrospective, descriptive study. We reviewed clinical records of patients who underwent surgery between January 2017 and November 2019. This study has been approved by the ethics committee of Arauco health service. Results: 77 tympanoplasties were performed, of which 56 ears met the inclusion criteria. $71.43 \%$ were female. The age range is 8 to 64 years. $41 \%$ had chronic pathologies. $51.79 \%$ were left ear surgeries. $92.86 \%$ were type I tympanoplasty. An endoauricular approach was performed in $58.93 \%$. A cartilage - perichondrium composite graft was used in 75\% of the surgeries. A medial technique was performed in $94.94 \%$. Anatomical success was achieved in $85.71 \%$ of patients. An average gain over $10 \mathrm{~dB}$ was obtained in $60.71 \%$ of the patients. Conclusion: There were no significant differences regarding the surgical choices with the anatomical and auditory results. Keywords: Chronic otitis media, tympanoplasty, tympanic membrane perforation.
\end{abstract}

\section{Introducción}

La otitis media crónica (OMC) es un motivo de consulta frecuente en otorrinolaringología, estando presente en todas las edades del ciclo vital $^{1-3}$. La OMC se define como una patología inflamatoria crónica de las estructuras del oído medio y mastoides de más de 3 meses de evolución ${ }^{4}$. Con respecto a la incidencia, una revisión sistemática que analizó 21 áreas 
regionales de la Organización Mundial de la Salud, determinó una incidencia global de la OMC de 4,76 por 1.000 habitantes, siendo la zona de Latinoamérica andina, la con más baja incidencia, de 1,7 por 1.000 habitantes ${ }^{5}$. La OMC es la principal causa de hipoacusia conductiva y, además, se puede asociar a mayor incidencia de hipoacusia neurosensorial según un estudio de Elrheem y cols ${ }^{6}$.

$\mathrm{La}$ OMC se puede clasificar en OMC simple y OMC colesteatomatosa. La simple se define como una perforación timpánica única, la colesteatomatosa presenta un colesteatoma, (acumulación de queratina proveniente de epitelio escamoso ubicado en el oído medio, contiene colagenasas y proteinasas que pueden destruir tejidos adyacentes). Este tipo de OMC puede subclasificarse en congénita y adquirida primaria o secundaria ${ }^{7}$.

El cuadro clínico clásico de la OMC simple se caracteriza por episodios recurrentes e indoloros de otorrea principalmente en relación a la entrada de agua o cuadros respiratorios altos. Al examen físico, en periodo de inactividad, se observa una perforación timpánica. Mientras que durante una fase activa se puede observar otorrea en el conducto auditivo y el oído medio $^{8}$. En este periodo se pueden encontrar bacterias aerobias (Pseudomonas aeruginosa, Escherichia coli, S. aureus, Streptococcus pyogenes, Proteus mirabilis, Klebsiella species) o anaerobias (Bacteroides, Peptostreptococcus, Proprionibacterium $)^{8-10}$.

Si bien gracias al advenimiento de la era antibiótica las complicaciones derivadas de la OMC han disminuido considerablemente, como por ejemplo una disminución de la mastoiditis de un 32\% a 6\% logrado ya en el año 1950, y por ende la mortalidad asociada a estas. En la actualidad aún existen casos con complicaciones fatales, estando descrita una mortalidad entre $2,4 \%$ a $13 \%$ relacionada a las complicaciones de la $\mathrm{OMC}^{11}$.

El tratamiento de elección en la OMC simple inactiva es la timpanoplastía. Esta consiste en reparar la membrana timpánica, con injertos autólogos, o sin injertos, como por ejemplo, al utilizar papel de arroz. Además, se puede agregar la reparación de la cadena osicular en caso de presentar alguna alteración con el objetivo de erradicar la enfermedad, prevenir la recurrencia y preservar o mejorar la audición ${ }^{12}$.
La primera timpanoplastía fue realizada por Berthold en 1878, pero no fue hasta 1952 que Wullstein y Zollner plantean los conceptos quirúrgicos actuales del procedimiento ${ }^{13,14}$. La timpanoplastía tiene diferentes clasificaciones, pero en Chile mantiene su clasificación inicial propuesta por Wullstein el año $1956^{15}$, agregándose una modificación según el tipo de osciculoplastía ${ }^{16}$ (Tabla 1).

El abordaje quirúrgico puede ser transcanal (o también denominado endoaural), endopreauricular o retroauricular. Al momento de posicionar el injerto se puede utilizar una técnica medial o underlay (injerto se posiciona en la cara medial del tímpano), una técnica lateral u overlay (injerto se posiciona en la cara lateral del tímpano), una técnica inlay (injerto se posiciona a nivel del remanente timpánico), o una técnica combinada en donde se aplica más de una técnica ${ }^{17}$. En cuanto al tipo de injerto, actualmente se utiliza un injerto autólogo. Este puede ser de fascia temporal, pericondrio y/o cartílago tragal o de concha auricular, de grasa, o combinados ${ }^{18}$. La fascia ha sido el material más comúnmente utilizado.

Tabla 1. Clasificación de Wüllstein modificada para timpanoplastias

- Tipo I

Revisión de cadena osicular con cadena osicular indemne

- Tipo /l

Alteración de martillo. Osiculoplastía con prótesis o injerto que se une al yunque

- Tipo III

- Clásica: Supraestructura del estribo presente, el injerto se apoya directo

- IIIA: Supraestructura del estribo presente. Se utiliza un injerto que se interpone entre la supraestructura del estribo y el neoptímpano. Puede ser maleoloestapediopexia o miringoestapediopexia - IIIB Estribo ausente, platina presente y móvil. Se utiliza un injerto que se interpone entre la platina del estribo y el neoptímpano. Puede ser maleoloplatinopexia o miringoplatinopexia

- Tipo IV

Platina expuesta con bolsa de aire entre ventana redonda e injerto

- Tipo $\mathrm{V}$

Ausencia de toda la cadena osicular. Platina fija. Ventana en conducto semicircular horizontal cubierta por injerto 
Diversos estudios comparan el uso de fascia y cartílago concluyendo en su mayoría que no hay diferencias significativas en cuanto a resultado anatómico y auditivo ${ }^{19-22}$. Destaca el estudio de Xabier y cols., quienes consideran al injerto de cartílago como una alternativa recomendada en casos de alto riesgo de reperforación, como en pacientes con disfunción tubaria, atelectasia timpánica, reperforaciones, entre otros ${ }^{23}$. Siguiendo este planteamiento, el estudio de Avani y cols., obtuvo diferencias significativas en cuanto a resultados anatómicos con el uso de cartílago ${ }^{24}$. El éxito anatómico se define como cierre completo de la perforación timpánica, este éxito es variable. Según distintas series internacionales van desde un $75 \%$ a un $98 \%$ de éxito. En cuanto a los resultados auditivos, los resultados son favorables entre un $82 \%$ y $95 \%{ }^{25-27}$.

En Chile no fue hasta el año 1956 que se publicó la primera revisión sobre indicaciones, técnicas y resultados asociados a la intervención $^{28}$. Desde entonces, y sobre todo en las últimas décadas, se han publicado múltiples trabajos con éxito anatómico y auditivo de entre un $79 \%$ y $90 \%$ y un $62 \%$ y $92 \%$ respectivamente ${ }^{29-33}$.

\section{Objetivo}

Realizar una descripción epidemiológica de los pacientes con diagnóstico de OMC simple sometidos a timpanoplastía en el Hospital San Vicente de Arauco entre los años 2017 y 2019.

\section{Material y Método}

Se realizó un estudio retrospectivo descriptivo. Se revisaron las fichas clínicas de todos los pacientes sometidos a timpanoplastía en el Hospital de Arauco entre enero de 2017 y diciembre de 2019.

\section{Criterios de inclusión}

Diagnóstico de otitis media crónica no colesteatomatosa, oído seco al menos 6 meses, audiometría preoperatoria, protocolo operatorio completo, audiometría posoperatoria, control de al menos 6 meses posterior a la cirugía.

\section{Criterios de exclusión}

Otitis media colesteatomatosa, timpanoplastías por reconstrucción funcional posterior a cirugía radical de oído, perforación timpánica traumática, timpanoplastía primaria con injerto de grasa, timpanoplastía secundaria (reintervención).

De los pacientes seleccionados se registró edad, sexo, antecedentes mórbidos, tipo de perforación, promedio de tonos puros (PTP) a los $500,1.000$ y $2.000 \mathrm{~Hz}$ pre y posoperatorios, técnica quirúrgica (tipo timpanoplastía, injerto y abordaje) y presencia de complicación posoperatoria. No se consideró el tono de 4000 $\mathrm{Hz}$ dado que en el promedio de PTP de las audiometrías de nuestro hospital no suelen incluir esta frecuencia.

Los resultados auditivos se clasificaron como buenos (más de $10 \mathrm{~dB}$ aéreos de ganancia sobre PTP preoperatorio), regulares (variación entre $+10 \mathrm{~dB}$ y $-10 \mathrm{~dB}$ del umbral preoperatorio) y malos (más de $10 \mathrm{~dB}$ de pérdida con relación al preoperatorio. Se utilizó estadística descriptiva para analizar los datos. Se cuenta con la aprobación del comité de ética de nuestro hospital.

\section{Resultados}

Durante el periodo en estudio se realizaron 77 timpanoplastías, de las cuales 56 cumplieron con los criterios de inclusión. Se sometieron a cirugía 50 pacientes y 56 oídos. Se observó que $74 \%(n=37)$ fueron del sexo femenino, $y$ un $26 \%(n=13)$ del sexo masculino. En total intervinieron bilateralmente 6 pacientes; 3 hombres y 3 mujeres.

Del total de pacientes un 32\% $(\mathrm{n}=16)$ tenían diagnóstico de OMC crónica bilateral. En cuanto a la edad, el rango va desde los 8 a los 64 años, la media aritmética fue de 40,9 años con una desviación estándar de $\pm 15,6$. En cuanto a los antecedentes mórbidos previos, $40 \%(n=20)$ presentaba alguna patología, dentro de las que destacan enfermedades de tipo cardiovascular como diabetes, hipertensión y/o dislipidemia en el 24\% $(\mathrm{n}=12)$. El $51,79 \%(n=29)$ corresponde a cirugías de oído izquierdo (Tabla 2).

De las cirugías realizadas el $92,86 \%(n=52)$ corresponde a timpanoplastía tipo I. Solo se 
realizaron 3 timpanoplastías tipo IIIa con uso de prótesis parcial (PORP) de titanio con longitud variable y 1 del tipo IIIb con uso de prótesis total (TORP) de titanio con longitud variable, correspondiente a un 5,36\% y $1,79 \%$ respectivamente.

Respecto a la técnica quirúrgica, se utilizó un abordaje endopreauricular el 58,93\% $(\mathrm{n}=33)$ de los casos, retroauricular en el $32,14 \%(\mathrm{n}=18)$ y transcanal en el $8,93 \%$ $(\mathrm{n}=5)$. El tipo de injerto utilizado fue predominantemente de tipo compuesto (cartílagopericondrio) en un $75 \%(n=42)$, seguido de fascia en un $14 \%(\mathrm{n}=8)$, mixto (cartílagofascia) en un $7 \%$ y dos pacientes solo con pericondrio correspondiente a un $4 \%$. Del total de operaciones se usó una técnica medial en un $94,94 \%(\mathrm{n}=53)$, una técnica lateral en un $3,57 \%$ y en solo un paciente $(1,79 \%)$ se utilizó técnica combinada inlay/overlay. De los pacientes operados con cartílago y pericondrio nueve fueron en empalizada (Tabla 3 ).

Se obtuvo un éxito anatómico en 48 procedimientos lo que corresponde a un $85,71 \%$ de los pacientes. La complicación más frecuente fue la reperforación timpánica, que alcanzó un $8,9 \%(n=5)$ seguida por la dehiscencia de injerto en un 3,6\% y, por último, retracción del injerto en un $1,8 \%(n=1)$. Del total de perforaciones un $6 \%(n=3)$ fueron perforaciones puntiformes y en su totalidad fueron sometidos a injerto de grasa (Tabla 4).

Si se contabiliza el éxito del injerto de grasa precoz (menos de 3 meses postimpanoplastía) como éxito, el total de perforaciones timpánicas que persistieron fueron 2 , equivalentes a un $3,57 \%$ del total de pacientes.

En cuanto al tipo de injerto utilizado se observó éxito anatómico de 36 de los 40 pacientes operados con injerto de cartílago-pericondrio (90\%). Al usar fascia se obtuvo un éxito de 75\% y al utilizar pericondrio o injerto compuesto (fascia-cartílago) el éxito corresponde a un $50 \%$ y $100 \%$ respectivamente (Tabla 4 ). Considerando la edad como variable, destaca que el éxito anatómico se obtuvo en el 100\% del grupo pediátrico y adolescente $(\mathrm{n}=5 \mathrm{y} \mathrm{n}=3$, respectivamente).

Al evaluar el éxito anatómico según la colocación del injerto, se evidenció un éxito anatómico en 45 de los 53 pacientes operados con técnica medial, lo que equivale al $84,91 \%$.

\begin{tabular}{|c|c|c|c|}
\hline \multicolumn{2}{|c|}{ Descripción demográfica } & \multirow{2}{*}{$\begin{array}{c}\text { n } \\
37\end{array}$} & \multirow{2}{*}{$\begin{array}{c}\% \\
74\end{array}$} \\
\hline Sexo & Femenino & & \\
\hline & Bilateral & 3 & \\
\hline & Masculino & 13 & 26 \\
\hline & Bilateral & 3 & \\
\hline \multirow[t]{3}{*}{ Edad } & Menor 15 años & 3 & 6 \\
\hline & $15-20$ años & 4 & 8 \\
\hline & Entre 20 y 65 años & 43 & 86 \\
\hline \multirow[t]{5}{*}{ Comorbilidad } & No & 30 & 60 \\
\hline & Sí & 20 & 40 \\
\hline & Cardiovascular & 12 & 24 \\
\hline & Tabaquismo & 6 & 12 \\
\hline & Otros & 2 & 4 \\
\hline \multirow[t]{2}{*}{ Lateralidad } & Izquierdo & 29 & 52 \\
\hline & Derecho & 27 & 48 \\
\hline
\end{tabular}

\begin{tabular}{llrr}
$\begin{array}{l}\text { Tabla 3. Resultados según tipo de timpanoplastía, } \\
\text { abordaje, técnica utilizada y tipo de injerto }\end{array}$ \\
\hline \multirow{4}{*}{$\begin{array}{l}\text { Tipo de } \\
\text { timpanoplastía }\end{array}$} & I & Illa & $\%$ \\
& Illb & 3 & 93 \\
Abordaje & Endoapreauricular & 33 & 59 \\
& Retroauricular & 18 & 32 \\
& Transcanal & 5 & 9 \\
\hline Técnica & Lateral - Overlay & 2 & 3 \\
& Medial - Underlay & 53 & 95 \\
& Mixta & 1 & 2 \\
Tipo de injerto & Cartílago-pericondrio & 42 & 75 \\
& Fascia temporal & 8 & 14 \\
& Cartílago-fascia & 4 & 7 \\
& Pericondrio & 2 & 4 \\
\hline
\end{tabular}

De los operados con técnica lateral el éxito se evidencia en el total de pacientes operados $(\mathrm{n}=2)$. El único paciente operado con técnica mixta obtuvo éxito anatómico, lo que representa el 100\% (Tabla 5).

El éxito anatómico según el tipo de timpanoplastía asciende al $86,5 \%(\mathrm{n}=45)$ en el tipo I, $66,67 \%(\mathrm{n}=2)$ en el tipo IIIa y en el $100 \%$ del tipo IIIb (Tabla 6). 
Tabla 4. Resultados anatómicos según tipo de injerto

\begin{tabular}{lcccc}
\hline & $\begin{array}{c}\text { Neotímpano indemne } \\
\%(\mathbf{n})\end{array}$ & $\begin{array}{c}\text { Reperforación } \\
\%(\mathbf{n})\end{array}$ & $\begin{array}{c}\text { Retracción } \\
\%(\mathbf{n})\end{array}$ & $\begin{array}{c}\text { Dehiscencia } \\
\%(\mathbf{n})\end{array}$ \\
\hline Cartílago pericondrio & $88,2 \%(37)$ & $7 \%(3)$ & $2,4 \%(1)$ & $2,4 \%(1)$ \\
\hline Fascia & $75 \%(6)$ & $25 \%(2)$ & $0 \%$ & $0 \%$ \\
\hline Cartílago fascia & $75 \%(3)$ & $0 \%$ & $0 \%$ & $25 \%(1)$ \\
\hline Pericondrio & $100 \%(2)$ & $0 \%$ & $0 \%$ & $0 \%$ \\
\hline Total de pacientes $\mathrm{n}(\%)$ & $48(85,7 \%)$ & $5(8,9 \%)$ & $1(1,8 \%)$ & $2(3,6 \%)$ \\
\hline
\end{tabular}

En el presente estudio no se encontró relación estadísticamente significativa entre los antecedentes demográficos, comorbilidades, técnica quirúrgica, tipo de timpanoplastía, tipo de injerto y el resultado anatómico final.

Con relación al resultado auditivo se obtuvo ganancia sobre $10 \mathrm{~dB}$ en un $60,71 \%$ $(n=34)$, entre $\pm 10 \mathrm{~dB}$ en el $37,5 \%$ (21) y una pérdida sobre $10 \mathrm{~dB}$ de audición en solo un $1,79 \%(\mathrm{n}=1$, Tabla 7$)$.

\begin{tabular}{|ccc|}
\hline \multicolumn{3}{l}{ Tabla 5. Resultado éxito anatómico según técnica } \\
quirúrgica \\
\hline Técnica quirúrgica & $\mathbf{n} /$ total & $\%$ \\
\hline Medial & $45 / 53$ & 84,91 \\
Lateral & $2 / 2$ & $100 \%$ \\
Mixta & $1 / 1$ & $100 \%$ \\
\hline
\end{tabular}

\section{Tabla 6. Resultado anatómico según tipo de} timpanoplastía

\begin{tabular}{ccc} 
Timpanoplastía & Éxito anatómico $\mathbf{n}$ & $\%$ \\
\hline IIIla & $45 / 52$ & 87 \\
IIIb & $2 / 3$ & 67 \\
\hline
\end{tabular}

\begin{tabular}{lcc|}
\hline Tabla 7. Resultado auditivo & & \\
\hline $\begin{array}{l}\text { Diferencia GAP óseo-aéreo } \\
\text { (ganancia) }\end{array}$ & $\mathbf{n}$ & $\%$ \\
\hline Mayor $10 \mathrm{~dB}$ & 34 & 60,71 \\
\hline Entre $\pm 10 \mathrm{~dB}$ & 21 & 37,5 \\
\hline Pérdida sobre $10 \mathrm{~dB}$ & 1 & 1,79 \\
\hline
\end{tabular}

\section{Discusión}

Del análisis de los datos recopilados en nuestro estudio, podemos decir que las características epidemiológicas de los pacientes intervenidos en nuestro servicio son comparables a los publicados en la literatura internacional y nacional ${ }^{19-22,25,26,34-36}$.

El grupo estudiado comprendió pacientes en edades infantiles y adultas, con un predominio en edades medias. Distintas series recomiendan la realización de una timpanoplastía desde los siete años de vida ${ }^{37}$. Sin embargo este criterio es difícil de aplicar de manera general en toda la población ya que depende del acceso a salud, escolaridad de los cuidadores, situación socioeconómica, entre otros. Es por esto que nosotros planteamos un criterio clínico de pasar al menos dos inviernos con una OMC inactiva para la realización de una timpanoplastía. Esto lo fundamentamos bajo el supuesto de que la función tubaria del paciente ha madurado. En el otro extremo, existe una tendencia a no operar pacientes sobre 65 años por un eventual menor éxito anatómico, sumado a que estos pacientes suelen presentar mayores comorbilidades y mayor morbimortalidad perioperatoria. Sin embargo, hoy en día gracias a los avances en salud, la expectativa de vida de los pacientes ha aumentado, como también la compensación y control de sus patologías de base y el acceso a tratamiento. Distintos estudios han analizado el grupo etario de más de 60 y 65 años encontrando un éxito anatómico similar a los grupos de menos de 59 años, existiendo solo una diferencia en el éxito auditivo, el cual disminuye en edades más avanzadas ${ }^{38,39}$. En nuestro estudio dejamos como límite arbitrario los 65 años de edad dado 
que la ruralidad de la zona no permite una compensación y control de estos pacientes de manera precoz, y por esto podrían presentar una mayor morbilidad posoperatoria.

Con relación a la técnica quirúrgica, la mayoría de los procedimientos se llevaron a cabo por la vía endopreauricular (59\%), con técnica medial (53\%) y uso de injerto cartílagopericondrio $(72 \%)$ como injerto. La elección del injerto puede estar determinada con la asociación que ha tenido a mayor estabilidad y rigidez, sobre todo en casos de perforaciones amplias, reperforaciones o atelectasias ${ }^{23,24}$. Además, la preferencia fundamental del uso de injerto combinado radica en nuestra ubicación geográfica, en donde nuestra zona presenta una ruralidad de un 32,3\% según la $\mathrm{OCDE}^{40}$, por lo que los pacientes deben desplazarse 1 a 4 horas desde zonas rurales para llegar a nuestro hospital. Si agregamos el bajo acceso a vehículos particulares, la necesidad de trabajo físico (cortar leña, cuidado del ganado, etc.), dificultad de acceso a urgencia, entre otros, el usar un injerto combinado nos da más seguridad de firmeza para el proceso de recuperación posoperatoria ambulatoria.

En nuestro estudio se obtuvo un éxito anatómico de un $85,7 \%$, que asciende a $91 \%$ si consideramos tímpano indemne posterior a injerto graso precoz ambulatorio. Esta diferenciación la planteamos en base que el injerto de grasa de realizó dentro de los primeros 3 meses de posoperatorio bajo anestesia local, significando un procedimiento ambulatorio sencillo para el paciente. El 97\% de los pacientes pudo mantener o mejorar su audición posterior al procedimiento.

Con respecto al tipo de timpanoplastía en su gran mayoría fueron de tipo I (93\%). En las timpanoplastías tipo IIIa se evidencian los peores resultados anatómicos (67\%). Esto debe ser interpretado con cautela considerando su número reducido.

\section{Conclusión}

La OMC es una patología frecuente en la consulta otorrinolaringológica y con ello la realización de timpanoplastía como tratamiento. En el presente estudio no se encontraron diferencias significativas importantes que relacionan las elecciones quirúrgicas con los resultados anatómicos y auditivos del procedimiento a mediano plazo.

\section{Bibliografía}

1. Ruz S, Breinbauer H, Arancibia M. Análisis epidemiológico de la patología otorrinolaringológica ambulatoria en el Hospital San Juan de Dios. Rev Otorrinolaringol Cir Cabeza Cuello. 2009;69(3):227232. doi: $10.4067 / S 0718-48162009000300004$.

2. Seymour C, Pardo J, Bahamonde H. Patología otorrinolaringológica ambulatoria en el Hospital Clínico de la Universidad de Chile durante el año 2008: Reporte de 9.157 pacientes. Rev Otorrinolaringol Cir Cabeza Cuello. 2011;71(3):237-240. doi: 10.4067/ S0718-48162011000300008.

3. Adoga A, Nimkur T, Silas O. Chronic suppurative otitis media: Socio-economic implications in a tertiary hospital in Northern Nigeria. Pan Afr Med J. 2010;4:3. doi: 10.4314/pamj.v4i1.53613.

4. Goycoolea MV, Hueb MM, Ruah C. Definitions and terminology. Otolaryngol Clin North America. 1991;24(4):757-761.

5. Monasta L, Ronfani L, Marchetti F, et al. Burden of disease caused by otitis media: systematic review and global estimates. PLoS One. 2012;7(4):e36226. doi: 10.1371/journal.pone.0036226.

6. Singer AEA, Abdel-Naby Awad OG, El-Kader RMA, Mohamed AR. Risk factors of sensorineural hearing loss in patients with unilateral safe chronic suppurative otitis media. Am J Otolaryngol. 2018;39(2):88-93. doi: 10.1016/j.amjoto.2018.01.002.

7. Bartel R, Cruellas F, Hamdan M, Benjumea F, Huguet G, González-Compta X, Cisa E, Manos M. Endoscopic type 3 tympanoplasty: Functional outcomes in chronic otitis media. Acta Otorrinolaringol Esp. 2020;71(2):83-87. doi: 10.1016/j. otorri.2019.02.001.

8. Bobby GW, Zadik P. Bacteriology of otitis media in Ghana. Trop Doct. 1987;17(2):91-2. doi: 10.1177/004947558701700216.

9. Brook I, Frazier E. Microbial dynamics of persistent purulent otitis media in children. J Pediatrics. 1996;128(2):237-240. doi: 10.1016/s00223476(96)70397-9.

10. Browning G, Gatehouse S, Calder T. Medical management of active chronic otitis media: a controlled study. J Laryngol Otol. 1988;102: 491-495. doi: 10.1017/S0022215100105444.

11. Baysal E, Erkutlu I, Mete A, Alptekin M, Oz A, Karataş ZA, Celenk F, Mumbuc S, Kanlikama M. Complications and treatment of chronic otitis media. J Craniofac Surg. 2013;24(2):464-7. doi: 10.1097/ SCS.0b013e318271046a. 
12. Merchant SN, Rosowski JJ, Mckenna MJ. Tympanoplasty. Operative Techniques in Otolaryngology, Head And Neck Surgery. 2003;14(4):224-236. doi: 10.1053/S10431810(03)00092-7.

13. Mudry A. History of Myringoplasty and Tympanoplasty Type I. Otolaryngology-Head and Neck Surgery. 2008;139(5):613-614. doi: 10.1016/j. otohns.2008.07.014.

14. Zollner F. The principles of plastic surgery of the sound-conducting apparatus. J Laryngol Otol. 1955; 69(10):637-52.

15. Wüllstein $\mathrm{H}$. Theory and practice of tympanoplasty. Laryngoscope. 1956; 66(8):1076-93. doi: 10.1288/00005537-195608000-00008.

16. Nazar G, Bustos C, Larraguibel S, Larach F, Iñiguez R. Timpanoplastía tipo III. Rev Otorrinolaringol Cir Cabeza Cuello. 2003;63:157-166.

17. Farrior JB. Incisions in tympanoplasty: anatomic considerations and indications. Laryngoscope. 1983;93(1):75-86. doi: 10.1288/00005537-19830100000015.

18. Boedts D. Tympanic grafting materials. Acta Otorhinolaryngol Belg. 1995;49(2):193-9.

19. Kazikdas KC, Onal K, Boyraz I, Karabulut E. Palisade cartilage tympanoplasty for management of subtotal perforations: a comparison with the temporalis fascia technique. Eur Arch Otorhinolaryngol. 2007;264(9):985-9. doi: 10.1007/s00405-007-0291-3.

20. Bayram A, Bayar Muluk N, Cingi C, Bafaqeeh SA. Success rates for various graft materials in tympanoplasty - A review. J Otol. 2020;15(3):107-111. doi: 10.1016/j.joto.2020.01.001.

21. Onal K, Arslanoglu S, Songu M, Demiray U, Demirpehlivan IA. Functional results of temporalis fascia versus cartilage tympanoplasty in patients with bilateral chronic otitis media. J Laryngol Otol. 2012;126(1):22-5. doi: 10.1017/S0022215111002817.

22. Sen A, Özdamar K. Which graft should be used for the pediatric transcanal endoscopic type 1 tympanoplasty? A comparative clinical study. Int J Pediatr Otorhinolaryngol. 2019;121:76-80. doi: 10.1016/j.ijporl.2019.02.046.

23. Altuna X, Navarro JJ. Miringoplastia con cartílago en isla. Resultados anatómicos y funcionales. Acta Otorrinolaringol Esp. 2020;61(2)100-105. doi: 10.1016/j.otorri.2009.09.002.

24. Jain A, Samdani S, Sharma MP, Meena V. Island cartilage vs temporalis fascia in type 1 tympanoplasty: A prospective study. Acta Otorrinolaringol Esp. 2018;69(6):311-317. doi: 10.1016/j.otorri.2017.10.004.

25. Dursun E, Demir E, Terzi S, Co kun ZÖ, Çeliker M, Erdivanlı ÖÇ. Bilateral same-day endoscopic tympanoplasty. Am J Otolaryngol. 2020;41(2):102397. doi: 10.1016/j.amjoto.2020.102397.

26. Shoman NM. Clinical and audiometric outcomes of palisade cartilage myringoplasty under local anesthetic in an office setting. Am J Otolaryngol. 2019;40(4):482-486. doi: 10.1016/j. amjoto.2019.03.015.

27. Demirpehlivan IA, Onal K, Arslanoglu S, Songu M, Ciger E, Can N. Comparison of different tympanic membrane reconstruction techniques in type I tympanoplasty. Eur Arch Otorhinolaryngol. 2011;268(3):471-4. doi: 10.1007/s00405-010-1473-y.

28. Maspetiol R. Indicaciones y técnica de a timpanoplastía (RR). Rev Otorrinolaringol Cir Cabeza Cuello.1956;16:17.

29. De la Paz F, Pardo J, Fernández R. Timpanoplastías en adultos en el Complejo Asistencial Dr. Sótero del Río (CASR): Revisión de 5 años. Rev Otorrinolaringol Cir Cabeza Cuello. 2009;69(3):221-226. doi: 10.4067/ S0718-48162009000300003.

30. Toro C, Naser A, Sanhueza C, Valdés C, Gormaz JP, Molina V, Leslie A. Timpanoplastías en adultos en el Hospital Clínico de la Universidad de Chile: Revisión de 10 años. Rev Otorrinolaringol Cir Cabeza Cuello. 2007;67(3):237-243. doi: 10.4067/S071848162007000300005.

31. Esquivel P, Naser A, Bustamante C. Timpanoplastía en niños: Experiencia de 10 años en el Hospital Clínico de la Universidad de Chile. Rev Otorrinolaringol Cir Cabeza Cuello. 2006;66(1):7-12. doi: 10.4067/S0718-48162006000100002.

32. Garfias R, Andrade T, Maul X, Baeza MA, Caro J. Timpanoplastía: Revisión y experiencia de 4 años en el Hospital Clínico de la Universidad Católica de Chile. Rev Otorrinolaringol Cir Cabeza Cuello. 2011;71(3):209-216. doi: 10.4067/S071848162011000300004.

33. Zelada U, Arregui R, Palacios E. Timpanoplastia en niños: experiencia de 10 años. Rev Otorrinolaringol Cir Cabeza Cuello. 2005;65:92-96.

34. Plodpai Y, Paje N. The outcomes of overlay myringoplasty: Endoscopic versus microscopic approach. Am J Otolaryngol. 2017;38(5):542-546. doi: 10.1016/j.amjoto.2017.05.007.

35. Salvador P, Gomes P, Silva F, Fonseca R. Type I Tympanoplasty: surgical success and prognostic factors. Acta Otorrinolaringol Esp ( $E n g l E d$ ). 2021;72(3):182-189. doi: 10.1016/j.otorri.2020.04.009.

36. Wang WH, Lin YC. Minimally invasive inlay and underlay tympanoplasty. Am J Otolaryngol. 2008;29(6):363-366. doi: 10.1016/j. amjoto.2007.11.002.

37. Ryan MA, Kaylie DM. What is the optimal age to repair tympanic membrane perforations in pediatric patients? Laryngoscope. 2016;126(10):2201-2202. doi: 10.1002/lary.26052.

38. Emmett JR. Age as a factor in the success of tympanoplasty: a comparison of outcomes in the young and old. Ear Nose Throat J. 1999;78(7):480483.

39. Demirci S, Tuzuner A, Callıglu EE, Akdaglı S, 
Karadas H, Caylan R. Tympanoplasty outcomes in elderly patients. Auris Nasus Larynx. 2016;43(1):3336. doi: 10.1016/j.anl.2015.05.002.

40. Publicación de la Oficina de Estudios y Políticas
Agrarias (Odepa) del Ministerio de Agricultura, Gobierno de Chile. Actualización septiembre de 2018. Recuperado de https://www.odepa.gob.cl/wpcontent/uploads/2017/08/Biobio.pdf. 